\title{
Exchange and Correlation Kernels at the Resonance Frequency: Implications for Excitation Energies in Density-Functional Theory
}

\author{
X. Gonze ${ }^{1,2}$ and M. Scheffler ${ }^{1}$ \\ ${ }^{1}$ Fritz-Haber-Institut der Max-Planck-Gesellshaft, Faradayweg 4-6, D-14195 Berlin-Dahlem, Germany \\ ${ }^{2}$ Unité PCPM, Université Catholique de Louvain, B-1348 Louvain-la-Neuve, Belgium
}

(Received 4 February 1999)

\begin{abstract}
Specific matrix elements of exchange and correlation kernels in time-dependent density-functional theory are computed. The knowledge of these matrix elements not only constrains approximate timedependent functionals. It also allows one to link different practical approaches to excited states, based either on density-functional theory or on many-body perturbation theory, despite the approximations that have been performed to derive them. [S0031-9007(99)09245-5]
\end{abstract}

PACS numbers: $31.50 .+\mathrm{w}, 71.10 .-\mathrm{w}, 71.35 .-\mathrm{y}$

Density-functional theory (DFT), as proposed by Hohenberg, Kohn, and Sham [1], has been highly successful in the analysis of interacting-particle ground states. Many efforts [2-11] have also been devoted to DFTbased schemes for excited states. The computation of excitation energies from DFT total-energy differences [2], a procedure known as $\triangle \mathrm{SCF}$, is formally restricted to transitions between the lowest states of different symmetries. The ensemble-density-functional theory [3], a more general path towards excitation energies, has not been used for actual calculations, to our knowledge. Recently, the feasibility of excitation-energy computations relying on two other, widely applicable, DFT-based formalisms has been demonstrated for atoms and small molecules. The first [4-9] starts from the extension of DFT to time-dependent (TD) phenomena [12-14] (TDDFT). The second $[10,11,15]$, due to Görling and Levy, builds a perturbation theory (GLPT) in the difference between the many-body and the second-quantized Kohn-Sham (KS) Hamiltonians, where the parameter of the perturbation is the coupling constant of the particle interaction, in such a way that, at each order, the exact density is recovered.

As an alternative to these DFT-based efforts, one may start from many-body perturbation theory and perform partial resummations of diagrams such as to build a screened interaction between dressed particles (quasiparticles) [16]. At the lowest order in the screened interaction, one obtains Hedin's $G W$ approximation [17] to quasiparticle energies (one particle is added or subtracted to the system), while the energy of excited states for which the number of particles is conserved can be deduced, in a subsequent step, from a Bethe-Salpeter (BS) equation describing the interaction between quasiparticles (e.g., electron-hole pairs). The application of these techniques to real materials is very demanding [18].

In the present Letter, we explore the relationships between excitation energies derived from time-dependent density-functional theory, the Görling-Levy perturbation theory, and the screened-interaction many-body perturbation theory. If excitation energies were derived without approximations, in the three formalisms, the results should be identical. Because of the specific theoretical developments, the most obvious simplifications are different, so that the practical schemes derived from these formalisms also differ. We find that some approximations used for practical calculations leave a connection between the approaches, at variance with the usual adiabatic local-density approximation (ALDA) in TDDFT [4-6], that leads to a different physical picture.

The expressions from different approaches are linked thanks to a new technique for computing selected elements of TD functional kernels at resonance, that is, at the frequencies corresponding to differences in KS eigenvalues, for which the independent-particle susceptibility of the KS system is resonant. It is first applied to the exact exchange kernel, whose matrix elements appear in a simplified TDDFT treatment of excitation energies based on a Laurent expansion, and are found identical to the first-order corrections to KS eigenvalues differences, in the GLPT. The knowledge of these matrix elements imposes a new constraint on approximate functionals. The same technique is then applied to an explicit exchangecorrelation (XC) functional that includes an approximate correlation contribution. It is shown that the corresponding Laurent-expansion TDDFT excitation energies can be split according to powers of the screened interaction, as in the $G W$-BS approach, and that they contain the $G W$ quasiparticle eigenvalue shifts.

For the sake of simplicity, we will consider systems made of spinless particles (or equivalently, fully spinpolarized systems) and focus on finite systems for which the energy levels of interest are nondegenerate (KS wave functions are real). The introduction of spin dependence and complex wave functions is technically straightforward, and does not modify the conclusions reached here. We use Hartree atomic units throughout. For integrals of the form $\int g_{1}\left(\mathbf{r}_{1}\right) f\left(\mathbf{r}_{1}, \mathbf{r}_{2}\right) g_{2}\left(\mathbf{r}_{2}\right) d \mathbf{r}_{1} d \mathbf{r}_{2}$, in which $g_{1}$ and $g_{2}$ are not wave functions, but densities or products of wave functions, we will use the notation $\left\{g_{1}|f| g_{2}\right\}$.

In TDDFT, the lowest-order Laurent expansion of the XC kernel and KS susceptibility, in the difference between the actual excitation frequency, $\Omega$, and the energy 
difference between corresponding KS eigenenergies, $\omega_{\nu}^{\mathrm{KS}}=\epsilon_{j}^{\mathrm{KS}}-\epsilon_{k}^{\mathrm{KS}}$, allows one to deduce approximate excitation energies as [5]

$\Omega \approx \omega_{\nu}^{\mathrm{KS}}+\left\{\Phi_{\nu}\left|V_{C}\right| \Phi_{\nu}\right\}+\left\{\Phi_{\nu}\left|f_{\mathrm{XC}}\left(\omega_{\nu}^{\mathrm{KS}}\right)\right| \Phi_{\nu}\right\}$.

The double index $\nu=(j, k)$ labels a single-particle transition from the occupied level $k$ to the unoccupied level $j, \Phi_{\nu}(\mathbf{r})$ is the product of $\mathrm{KS}$ wave functions $\phi_{k}(\mathbf{r}) \phi_{j}(\mathbf{r})$, $V_{C}$ is the Coulomb interaction kernel $\left(\frac{1}{\left|\mathbf{r}_{1}-\mathbf{r}_{2}\right|}\right)$, and $f_{\mathrm{XC}}\left(\mathbf{r}_{1}, \mathbf{r}_{2} ; \omega\right)$ is the frequency-dependent $\mathrm{XC}$ kernel.

In Eq. (1), the diagonal matrix elements of the Coulomb and $\mathrm{XC}$ kernel for the transition between occupied and unoccupied states correct the difference in KS eigenvalues. The Coulomb kernel gives an exchangelike interaction between the KS electron and hole $\left[\phi_{k}\left(\mathbf{r}_{1}\right) \phi_{j}\left(\mathbf{r}_{1}\right) \frac{1}{\left|\mathbf{r}_{1}-\mathbf{r}_{2}\right|} \times\right.$ $\left.\phi_{k}\left(\mathbf{r}_{2}\right) \phi_{j}\left(\mathbf{r}_{2}\right)\right]$. The XC kernel is easy to evaluate in the
ALDA (that ignore the functional dependence of the $\mathrm{XC}$ potential $V_{\mathrm{XC}}$ on densities at different times as well as different locations): It induces only a local, attractive, static electron-hole interaction $\left[\phi_{k}\left(\mathbf{r}_{1}\right) \phi_{j}\left(\mathbf{r}_{1}\right) \delta\left(\mathbf{r}_{1}-\mathbf{r}_{2}\right) \times\right.$ $\left.\frac{d V_{\mathrm{XC}}}{d n}\left(\mathbf{r}_{1}\right) \phi_{k}\left(\mathbf{r}_{2}\right) \phi_{j}\left(\mathbf{r}_{2}\right)\right]$. Then, as detailed by Cassida [4], quasiparticle energies (i.e., excitation energies, to which quasiparticle interactions make no contribution, such as ionization energies) are identified with unmodified KS energies [19].

Unlike the ALDA, we evaluate exactly the exchange contribution. Since we will use later the same technique for the correlation contribution, we treat them now on the same footing. The Keldysh formalism, as applied recently to the TDDFT case by van Leeuwen [13] (we follow his notations), leads to the following explicit expression for the TD kernel, second derivative of the action $A_{\mathrm{XC}}$, in the temporal (pseudotime) domain:

$$
\begin{aligned}
f_{\mathrm{XC}}\left(\mathbf{r}_{1} \tau_{1} ; \mathbf{r}_{2} \tau_{2}\right)= & \iint_{C} d t_{3} d \mathbf{r}_{3} d t_{4} d \mathbf{r}_{4} \frac{\delta u\left(\mathbf{r}_{3} \tau_{3}\right)}{\delta n\left(\mathbf{r}_{1} \tau_{1}\right)} \frac{\delta u\left(\mathbf{r}_{4} \tau_{4}\right)}{\delta n\left(\mathbf{r}_{2} \tau_{2}\right)} \\
& \times\left[\frac{\delta^{2} A_{\mathrm{XC}}}{\delta u\left(\mathbf{r}_{3} \tau_{3}\right) \delta u\left(\mathbf{r}_{4} \tau_{4}\right)}-\int_{C} d t_{5} d \mathbf{r}_{5} u_{\mathrm{XC}}\left(\mathbf{r}_{5} \tau_{5}\right) \frac{\delta^{2} n\left(\mathbf{r}_{5} \tau_{5}\right)}{\delta u\left(\mathbf{r}_{3} \tau_{3}\right) \delta u\left(\mathbf{r}_{4} \tau_{4}\right)}\right] .
\end{aligned}
$$

In this equation $u$ and $u_{\mathrm{XC}}$, respectively, denote the time-domain $\mathrm{KS}$ potential and the XC potential.

This general expression can be specialized to the TD linear response of an otherwise static system, in the frequency domain, and inserted in the integral needed in Eq. (1):

$$
\left\{\Phi_{\nu}\left|f_{\mathrm{XC}}(\omega)\right| \Phi_{\nu}\right\}=\left\{\Phi_{\nu}\left|\chi_{0}^{-1}(-\omega)\left[\frac{\delta^{2} A_{\mathrm{XC}}(\omega=0)}{\delta v_{s}(-\omega) \delta v_{s}(\omega)}-\int d \mathbf{r}_{5} v_{\mathrm{XC}}\left(\mathbf{r}_{5}\right) \frac{\delta^{2} n\left(\mathbf{r}_{5} ; \omega=0\right)}{\delta v_{s}(-\omega) \delta v_{s}(\omega)}\right] \chi_{0}^{-1}(\omega)\right| \Phi_{\nu}\right\}
$$

Note the appearance of the (static) XC potential $v_{\mathrm{XC}}(\mathbf{r})$. $\delta v_{s}(\mathbf{r} ; \omega)$ is a change of KS potential in the frequency domain, $\chi_{0}\left(\mathbf{r}_{1}, \mathbf{r}_{2} ; \omega\right)$ is the independent-particle susceptibility of the KS system, and $\chi_{0}^{-1}$ is the effective inverse of $\chi_{0}$, defined on a restricted space excluding constant functions [8]. The spatial dependence of potentials and susceptibilities is implicit in Eq. (3), as well as most integrals over spatial arguments. $A_{\mathrm{XC}}(\omega=0)$ and $n(\mathbf{r} ; \omega=0)$ are the time-independent part of the action integrand and the density, respectively [20].

When evaluated at $\omega=\omega_{\nu}^{\mathrm{KS}}$, Eq. (3) gives the XC kernel contribution to $\Omega$ in Eq. (1). This requires the inversion of $\chi_{0}$ around the frequency $\omega_{\nu}^{\mathrm{KS}}$, and its subsequent application to $\Phi_{\nu}(\mathbf{r})$. The explicit expression of $\chi_{0}$ is well known [see Eq. (14) of Ref. [8]] and exhibits a resonance at that frequency. The corresponding pole can be singled out before performing the inversion: $\chi_{0}\left(\mathbf{r}_{1}, \mathbf{r}_{2} ; \omega\right)=$ $\frac{\Phi_{\nu}\left(\mathbf{r}_{1}\right) \Phi_{\nu}\left(\mathbf{r}_{2}\right)}{\omega-\omega_{\nu}^{K S}}+\chi_{\nu}\left(\mathbf{r}_{1}, \mathbf{r}_{2} ; \omega\right)$, where the nonresonant contribution $\chi_{\nu}$ is implicitly defined by this equation. The Sherman-Morrison formula [21] allows the analytical inversion of such a decomposition. The application of $\chi_{0}^{-1}$ to $\Phi_{\nu}$, denoted $v_{\nu}$, gives

$$
\begin{aligned}
v_{\nu}(\omega) & \left.=\chi_{0}^{-1}(\omega) \mid \Phi_{\nu}\right\} \\
& =\left(\omega-\omega_{\nu}^{\mathrm{KS}}\right) \frac{\left.\chi_{\nu}^{-1}(\omega) \mid \Phi_{\nu}\right\}}{\left(\omega-\omega_{\nu}^{\mathrm{KS}}\right)-\left\{\Phi_{\nu}\left|\chi_{\nu}^{-1}(\omega)\right| \Phi_{\nu}\right\}} .
\end{aligned}
$$

A similar expression involving also $\omega-\omega_{\nu}^{\mathrm{KS}}$ can be derived in the case of $v_{\nu}(-\omega)$. The presence of the factor $\left(\omega-\omega_{\nu}^{\mathrm{KS}}\right)$ emphasizes the antiresonant behavior of $\chi_{0}^{-1}$. This characteristic of Eq. (4) allows one to extract the leading order of the frequency expansion of Eq. (3) around $\omega_{\nu}^{\mathrm{KS}}$, for XC actions whose explicit expression is known as a function of the KS potential through KS wave functions. Indeed, after technically straightforward calculations within perturbation theory (up to second-order wave functions), one finds

$$
\begin{aligned}
& \left\{v_{\nu}(-\omega)\left|\frac{\delta^{2} n(\mathbf{r} ; \omega=0)}{\delta v_{s}(-\omega) \delta v_{s}(\omega)}\right| v_{\nu}(\omega)\right\} \\
& \quad=\phi_{j}(\mathbf{r}) \phi_{j}(\mathbf{r})-\phi_{k}(\mathbf{r}) \phi_{k}(\mathbf{r})+\mathcal{O}\left(\omega-\omega_{\nu}^{\mathrm{KS}}\right),
\end{aligned}
$$

and, using the explicit exchange action of Ref. [13],

$$
\begin{aligned}
\left\{v_{\nu}(-\omega) \mid\right. & \left.\frac{\delta^{2} A_{x}(\omega=0)}{\delta v_{s}(-\omega) \delta v_{s}(\omega)} \mid v_{\nu}(\omega)\right\} \\
= & \left\langle\phi_{j}\left|\hat{v}_{x}^{\mathrm{HF}}\right| \phi_{j}\right\rangle-\left\langle\phi_{k}\left|\hat{v}_{x}^{\mathrm{HF}}\right| \phi_{k}\right\rangle-\left\{n_{k}\left|V_{C}\right| n_{j}\right\} \\
& +\mathcal{O}\left(\omega-\omega_{\nu}^{\mathrm{KS}}\right),
\end{aligned}
$$

where $\hat{v}_{x}^{\mathrm{HF}}$ is the Fock nonlocal operator evaluated with KS wave functions, and $n_{k}(\mathbf{r})$ is the square of KS wave function $\phi_{k}(\mathbf{r})$. Combining Eqs. (3), (5), and (6), we derive the following matrix element of the exchange kernel at resonance (in the limit of $\omega \rightarrow \omega_{\nu}^{\mathrm{KS}}$ ), valid for all pairs $(j, k)$ of unoccupied and occupied levels associated with a 
composite index $\nu$ :

$$
\begin{aligned}
\left\{\Phi_{\nu}\left|f_{x}\left(\omega_{\nu}^{\mathrm{KS}}\right)\right| \Phi_{\nu}\right\}= & \left\langle\phi_{j}\left|\hat{v}_{x}^{\mathrm{HF}}-v_{x}\right| \phi_{j}\right\rangle \\
& -\left\langle\phi_{k}\left|\hat{v}_{x}^{\mathrm{HF}}-v_{x}\right| \phi_{k}\right\rangle \\
& -\left\{n_{k}\left|V_{C}\right| n_{j}\right\} .
\end{aligned}
$$

This explicit expression for selected matrix elements of the TD exchange kernel provides, as such, a new constraint on approximate TD exchange functionals. Note the drastic simplification from the general matrix elements of the TD exchange kernel derived by Görling [8]. The combination of Eqs. (1) and (7), neglecting the correlation contribution, gives

$$
\begin{aligned}
\Omega \approx & \omega_{\nu}^{\mathrm{KS}}+\left\langle\phi_{j}\left|\hat{v}_{x}^{\mathrm{HF}}-v_{x}\right| \phi_{j}\right\rangle-\left\langle\phi_{k}\left|\hat{v}_{x}^{\mathrm{HF}}-v_{x}\right| \phi_{k}\right\rangle \\
& +\left\{\Phi_{\nu}\left|V_{C}\right| \Phi_{\nu}\right\}-\left\{n_{k}\left|V_{C}\right| n_{j}\right\} .
\end{aligned}
$$

This expression is identical to the one obtained in the first-order GLPT [see Eq. (A3) of Ref. [11], and also Ref. [10]]: Excitation energies from the TDDFT with exact treatment of the exchange kernel, in the Laurent approximation, and from first-order GLPT are equal [22].

The KS eigenenergies $\epsilon_{j}^{\mathrm{KS}}$ and $\epsilon_{k}^{\mathrm{KS}}$ are expectation values of the KS Hamiltonian that includes the exchange potential. Hence, the two expectation values of the difference between the Fock operator and the exchange potential in Eq. (8) induce a shift of KS energies $\epsilon_{j}^{\mathrm{KS}}$ and $\epsilon_{k}^{\mathrm{KS}}$, present in $\omega_{\nu}^{\mathrm{KS}}$, in the direction of the Hartree-Fock quasiparticle energies. So, unlike in the ALDA approach, the quasiparticle energies from TDDFT with exact treatment of the exchange kernel are shifted from the KS eigenenergies. The fifth term in Eq. (8) describes an unscreened Coulomb electron-hole attraction, to be contrasted with the effective interaction present in ALDA. However, the fourth term in Eq. (8) is the same exchangelike interaction as in the ALDA case, present in Eq. (1).

The dynamical treatment of correlation is more difficult than that of exchange. We will not pursue the investigation of GLPT that would involve higher-order terms. However, we will establish a link between TDDFT and the screened-interaction many-body perturbation theory. At variance with the decoupling between $G W$ and BS procedures, TDDFT computes the excitation energies in one operation. This might be a crucial advantage for practical applications. Our aim will be to show, at the adequate approximation levels, that TDDFT contains the ingredients characteristic of the $G W$ approach, and to point out how it accounts for the additional BS terms.

The $G W$ quasiparticle shift, evaluated in a non-selfconsistent, diagonal approximation, based on KS eigenenergy and eigenfunctions, leads to the following excitation energies:

$$
\begin{aligned}
\Omega \approx & \omega_{\nu}^{\mathrm{KS}}+\left\langle\phi_{j}\left|\Sigma^{G W}\left(\epsilon_{j}^{\mathrm{KS}}\right)-v_{\mathrm{XC}}\right| \phi_{j}\right\rangle \\
& -\left\langle\phi_{k}\left|\Sigma^{G W}\left(\epsilon_{k}^{\mathrm{KS}}\right)-v_{\mathrm{XC}}\right| \phi_{k}\right\rangle .
\end{aligned}
$$

$\Sigma^{G W}$ is the self-energy operator in the $G W$ approximation:

$$
\begin{aligned}
\Sigma^{G W}\left(\mathbf{r}_{1}, \mathbf{r}_{2} ; \boldsymbol{\epsilon}\right)= & \int \frac{d \boldsymbol{\epsilon}^{\prime}}{2 \pi} e^{-i \delta \epsilon^{\prime}} i G\left(\mathbf{r}_{1}, \mathbf{r}_{2} ; \epsilon-\boldsymbol{\epsilon}^{\prime}\right) \\
& \times W\left(\mathbf{r}_{1}, \mathbf{r}_{2} ; \boldsymbol{\epsilon}^{\prime}\right),
\end{aligned}
$$

where the Green's function $G$ is built from KS wave functions, and $W$ is the dynamically screened Coulomb interaction $\left(W=\varepsilon_{\mathrm{RPA}}^{-1} V_{C}\right.$, with $\varepsilon_{\mathrm{RPA}}=1-V_{C} \chi_{0}$ being the RPA dielectric screening). The $\epsilon^{\prime}$ integral can be further transformed to a contour integral in the complex plane.

In Eq. (9), the difference between KS eigenenergies is shifted by expectation values of the difference between the self-energy operator and the XC potential. The neglected BS corrections would make quasiparticles interact through an unscreened exchangelike term [compare with Eq. (1)] as well as through a screened Coulomb interaction. Excitonic effects would be described at that level. The screened Coulomb interaction is to be contrasted with the effective, local, electron-hole interaction of ALDA-TDDFT, and the unscreened Coulomb interaction of first-order GLPT.

A formally exact correlation functional can be obtained by the combination of the adiabatic connection method (based on a coupling constant integral) and the fluctuationdissipation theorem [23]. In the context of the DFT computation of van der Waals energy, Dobson [23] proposed a simplified expression,

$$
E_{C}=\int_{0}^{1} d \lambda \int_{0}^{\infty} \frac{d u}{2 \pi} \operatorname{Tr}\left\{V_{C}\left[\chi_{0}(i u)-\chi_{\mathrm{RPA}, \lambda}(i u)\right]\right\},
$$

in which the true susceptibility present in the exact $E_{C}$ expression is replaced by the RPA one, although the integral over the coupling constant $\lambda$ is retained.

The RPA susceptibility is a simple functional of $\chi_{0}$ $\left[\chi_{\mathrm{RPA}, \lambda}=\chi_{0}\left(1-\lambda V_{C} \chi_{0}\right)^{-1}\right]$. This latter quantity is a functional of the KS potential, itself a functional of the density. We can thus compute the corresponding correlation potential and kernel. Thanks to an integration by parts, we obtain that the correlation potential does not exhibit the integration over the coupling constant $\lambda$ :

$$
\begin{aligned}
v_{C}\left(\mathbf{r}_{1}\right)= & \frac{\delta E_{C}}{\delta n\left(\mathbf{r}_{1}\right)}=\int d \mathbf{r}_{2} \chi_{0}^{-1}\left(\mathbf{r}_{1}, \mathbf{r}_{2} ; \omega=0\right) \int_{0}^{\infty} \frac{d u}{2 \pi} \\
& \times \operatorname{Tr}\left\{\left[V_{C}-W(i u)\right] \frac{\delta \chi_{0}(i u)}{\delta v_{s}\left(\mathbf{r}_{2}\right)}\right\},
\end{aligned}
$$

where the same screened interaction $W$ as in the $G W$ approximation appears. For the computation of excited states, we generalize the correlation functional Eq. (10) to a TD action, and, neglecting a term in the second power of the screened interaction, derive

$$
\begin{aligned}
& \frac{\delta^{2} A_{C}(\omega=0)}{\delta v_{s}\left(\mathbf{r}_{1} ;-\omega\right) \delta v_{s}\left(\mathbf{r}_{2} ; \omega\right)} \approx \int_{0}^{\infty} \frac{d u}{2 \pi} \\
& \times \operatorname{Tr}\left\{\left[V_{C}-W(i u)\right] \frac{\delta^{2} \chi_{0}(i u)}{\delta v_{s}\left(\mathbf{r}_{1} ;-\omega\right) \delta v_{s}\left(\mathbf{r}_{2} ; \omega\right)}\right\} .
\end{aligned}
$$

For comparison with the $G W$ approximation, it is crucial that the coupling constant is absent, as in Eq. (11). The second derivative of $\chi_{0}$ with respect to TD changes of potential can also be evaluated (perturbation theory up to 
third-order wave functions), close to the resonance:

$$
\begin{aligned}
\left\{v_{\nu}(-\omega)\left|\frac{\delta^{2} \chi_{0}\left(\mathbf{r}_{1}, \mathbf{r}_{2} ; i u\right)}{\delta v_{s}(-\omega) \delta v_{s}(\omega)}\right| v_{\nu}(-\omega)\right\}= & \phi_{j}\left(\mathbf{r}_{1}\right) 2 \operatorname{Re}\left[G\left(\mathbf{r}_{1}, \mathbf{r}_{2} ; \epsilon_{j}^{\mathrm{KS}}+i u\right)\right] \phi_{j}\left(\mathbf{r}_{2}\right) \\
& -\phi_{k}\left(\mathbf{r}_{1}\right) 2 \operatorname{Re}\left[G\left(\mathbf{r}_{1}, \mathbf{r}_{2} ; \epsilon_{k}^{\mathrm{KS}}+i u\right)\right] \phi_{k}\left(\mathbf{r}_{2}\right)+\mathcal{O}\left(\omega-\omega_{\nu}^{\mathrm{KS}}\right),
\end{aligned}
$$

where the same Green's function $G$ as in the $G W$ approximation appears. The combination of Eqs. (12) and (13), needed in Eq. (3), gives the difference between an unscreened $G V_{C}$ term and a $G W$ term. We introduce this result in Eq. (1), and find that the main contribution of the first term $\left(G V_{C}\right)$ cancels the unscreened Fock operator that shifts the KS eigenvalues in Eq. (8).

We can now discuss the link between $G W$-BS excitation energies and TDDFT excitation energies obtained from an exact exchange kernel and an approximate correlation kernel: (i) As expected, the crucial $G W$ terms are contained in Eq. (1); (ii) the inclusion of correlation in TDDFT transforms the unscreened single-orbital matrix elements of Eq. (8) to dynamically screened matrix elements, as in Eq. (9); (iii) the subtraction of the same matrix elements of $v_{\mathrm{XC}}$ appear in both schemes, compare Eqs. (3)-(5) to Eq. (9); (iv) however, the contour integral deduced from Eq. (12) is shifted from the contour integral in the $G W$ approximation, and includes contributions from additional poles; (v) a term in the second power of the screened interaction has been neglected from Eq. (10) to Eq. (12); (vi) the RPA susceptibility was used in Eq. (10). Points (iv)-(vi) connect to BS as well as $\mathcal{O}\left(W^{n}\right)$ (with $n \geq 2$ ) corrections to Eq. (9). Understanding point (iv) also requires a careful analysis of our TD correlation action (especially the contour integral to be used with the fluctuation-dissipation theorem). A more detailed discussion of these aspects will be left for a future publication.

In this paper, we have examined the time-dependent kernels deduced from the exact exchange functional and an approximate correlation functional, at resonance. Links between approximate expressions for excitation energies from time-dependent DFT, the Görling-Levy perturbation theory, and Hedin's $G W$ approximation have been found: The difference between Kohn-Sham eigenvalues is corrected by one-particle shifts, followed by (unscreened or screened) interaction effects. We also noted that in the adiabatic local-density approximation to time-dependent DFT, no one-particle shift is present, and the link with the two other techniques is lost.

We thank A. Schindlmayr for a careful reading of the manuscript. X.G. acknowledges financial support from the A. von Humboldt foundation and from the National Fund for Scientific Research, Belgium.

[1] P. Hohenberg and W. Kohn, Phys. Rev. 136, B864 (1964); W. Kohn and L. J. Sham, Phys. Rev. 140, A1133 (1965).

[2] O. Gunnarsson and B. I. Lundqvist, Phys. Rev. B 13, 4274 (1976); T. Ziegler, A. Rauk, and B.J. Baerends, Theor.
Chim. Acta 43, 261 (1977); O. Gunnarsson and R. O. Jones, J. Chem. Phys. 72, 5357 (1980).

[3] A. Theophilou, J. Phys. C 12, 5419 (1979).

[4] M.E. Casida, in Recent Developments and Applications of Modern Density Functional Theory, edited by J.M. Seminario (Elsevier, Amsterdam, 1996).

[5] M. Petersilka, U. J. Gossman, and E. K. U. Gross, Phys. Rev. Lett. 76, 1212 (1996).

[6] See M.E. Casida et al., J. Chem. Phys. 108, 4439 (1998), and references therein.

[7] A. Görling, Phys. Rev. B 57, 3433 (1998).

[8] A. Görling, Int. J. Quantum Chem. 69, 265 (1998).

[9] S. J.A. van Gisbergen et al., Phys. Rev. A 57, 2556 (1998).

[10] A. Görling, Phys. Rev. A 54, 3912 (1996).

[11] C. Filippi, C. J. Umrigar, and X. Gonze, J. Chem. Phys. 107, 9994 (1997).

[12] E. Runge and E. K. U. Gross, Phys. Rev. Lett. 52, 997 (1984); see Ref. [4] for recent references.

[13] R. van Leeuwen, Phys. Rev. Lett. 80, 1280 (1998).

[14] J. F. Dobson, M. J. Bunner, and E. K. U. Gross, Phys. Rev. Lett. 79, 1905 (1997); G. Vignale, C. A. Ullrich, and S. Conti, Phys. Rev. Lett. 79, 4878 (1997); P. Hessler, J. Park, and K. Burke, Phys. Rev. Lett. 82, 378 (1999).

[15] A. Görling and M. Levy, Phys. Rev. B 47, 13105 (1993); Int. J. Quantum Chem. 29, 93 (1995).

[16] L. Hedin and S. Lundqvist, in Solid State Physics, edited by H. Ehrenreich, F. Seitz, and D. Turnbull (Academic Press, New York, 1969), Vol. 23, p. 1.

[17] L. Hedin, Phys. Rev. 139, A796 (1965).

[18] L. X. Benedict, E. L. Shirley, and R. Bohn, Phys. Rev. B 57, R9385 (1998); Phys. Rev. Lett. 80, 4514 (1998); S. Albrecht et al., Phys. Rev. Lett. 80, 4510 (1998); M. Rohlfing and S. Louie, Phys. Rev. Lett. 81, 2312 (1998).

[19] In the complex version of Eq. (1) — needed for scattering states - if one of the wave functions is delocalized, within ALDA, both Coulomb and XC contributions vanish.

[20] Although time independent, they are modified by a TD perturbation, in the same way as an oscillating current induces a constant potential drop in a diode, a phenomenon known as rectification. Albeit not apparent in our notation, perturbations in the frequency domain are switched on adiabatically, as in Ref. [8].

[21] See W.H. Press, B.P. Flannery, S. A. Teukolsky, and W.T. Vetterling, in Numerical Recipes: The Art of Scientific Computing (Cambridge University, New York, 1989), p. 66.

[22] For the specific case of two-electron systems, this equality had been noticed (p. 9999 of Ref. [11]; p. 2565 of Ref. [9]), but it was not suspected to hold in general.

[23] J.F. Dobson, in Topics in Condensed Matter Physics, edited by M.P. Das (Nova, New York, 1994); J.F. Dobson and B. Dinte, Phys. Rev. Lett. 76, 1780 (1996). 